\title{
Analysis of the Effect of Trastuzumab Combined with Docetaxel on Serum Tumor Markers in the Treatment of HER-2 Positive Breast Cancer and Factors Influencing Therapeutic Efficacy
}

\author{
Qi-Zhu Feng ${ }^{1-3}$ \\ Xian-Zhi Chen ${ }^{2}$ \\ Jie Sun ${ }^{4}$ \\ Man-Man Lu ${ }^{5}$ \\ Yong Wang ${ }^{6}$ \\ Qi Wang ${ }^{4}$ \\ Chao Zhang (D) ${ }^{1,3}$
}

'Department of General Surgery, First Affiliated Hospital of Anhui Medical University, Hefei, 230032, Anhui, People's Republic of China; ${ }^{2}$ Department of Breast Surgery, First Affiliated Hospital of Anhui University of Science and Technology (Huainan First People's Hospital), Huainan, 232007, Anhui, People's Republic of China; ${ }^{3}$ School of Continuing Education, Anhui Medical University, Hefei, 230032, Anhui, People's Republic of China; ${ }^{4}$ Department of General Surgery, First Affiliated Hospital of Anhui University of Science and Technology (Huainan First People's Hospital), Huainan, 232007, Anhui, People's Republic of China; ${ }^{5}$ Department of Laboratory Medicine, First Affiliated Hospital of Anhui University of Science and Technology (Huainan First People's Hospital), Huainan, 232007, Anhui, People's Republic of China; ${ }^{6}$ Department of Medical Oncology, First Affiliated Hospital of Anhui University of Science and Technology (Huainan First People's Hospital), Huainan, 232007, Anhui, People's Republic of China

Correspondence: Chao Zhang Department of General Surgery, First Affiliated Hospital of Anhui Medical University, Hefei, 230032, Anhui, People's Republic of China

Tel +86-I3956053990

Email zhangchaolove88@I63.com
Objective: To explore the influence of trastuzumab (TZ) combined with docetaxel (DTX) on serum tumor markers (TMs) in the treatment of human epidermal growth factor receptor 2-positive (HER-2+) breast cancer (BC) and to analyze the factors influencing therapeutic efficacy.

Methods: Ninety-six patients with HER-2+ BC treated in the First Affiliated Hospital of Anhui University Of Science and Technology from January 2019 to December 2020 were selected. According to different treatment plans, the patients were divided into two arms with 48 cases each. The control group (CG) was treated with DTX, and the research group (RG) was given TZ combined with DTX (TZ+DTX). The two arms were compared regarding the following aspects: curative effects, adverse reaction, alterations of TMs and inflammatory factors (IFs), and quality of life. Logistic regression analysis was performed to analyze the factors affecting the efficacy of patients.

Results: After treatment, the TMs carcinoembryonic antigen (CEA), carbohydrate antigen (CA)125 and CA15-3 were significantly lower in RG compared with CG. The levels of IFs C-reactive protein (CRP) and tumor necrosis factor- $\alpha$ (TNF- $\alpha$ ) were also lower in CG. The overall response rate and the Karnofsky performance status (KPS) score were significantly higher in RG. No evident difference was observed in the total incidence of adverse reactions between the two arms. The high expression of CEA, CA125 and CA15-3 as well as DTX monotherapy increased the risk of adverse prognosis.

Conclusion: TZ+DTX can effectively improve the clinical efficacy of HER-2+ BC patients and reduce their levels of serum TMs and IFs.

Keywords: trastuzumab combined with docetaxel, HER-2+ breast cancer, tumor markers, analysis of therapeutic factors

\section{Introduction}

Clinically, breast cancer (BC) is a common and frequently-occurring disease as well as one of the commonly seen malignancies in women. ${ }^{1}$ With the development of economy and the change of people's lifestyle, the incidence of $\mathrm{BC}$ is increasing in recent years. ${ }^{2}$ It has been reported that $20-30 \%$ of BC patients are human epidermal growth factor receptor 2 positive (HER-2+). ${ }^{3}$ It is reported that the expression of HER-2 gene is closely related to the degree of malignancy and disease progression of $\mathrm{BC}$ patients; it is also an important factor affecting the therapeutic effect, as well 
as one of the risk factors leading to adverse prognosis in $\mathrm{BC}$ patients. ${ }^{4}$ Therefore, finding suitable and effective intervention methods is particularly important for improving the prognosis of patients with HER-2+ BC.

Trastuzumab (TZ), a monoclonal targeted anti-cancer drug that specifically acts on HER-2 receptors, can bind to HER-2 and block the promoting effect of epidermal growth factor on tumor cell growth, thus improving the progression-free survival of patients. ${ }^{5} \mathrm{TZ}$ can also eliminate cancer cells by stimulating immune cells, and suppress the growth-enhancing effect of the HER-2 family by downregulating HER-2 gene. ${ }^{6}$ Therefore, TZ is highly targeted for patients with HER-2+ BC. ${ }^{7}$ However, clinical evidence shows that TZ alone is difficult to achieve satisfactory results, and will lead to a series of toxic and side effects such as rash, gastrointestinal reaction and bone marrow suppression in most patients during treatment. ${ }^{8}$ Studies have shown that TZ has been used to inhibit HER-2 activity, but the major resistance of $\mathrm{TZ}$ challenges its application in the treatment of HER-2+ BC; Therefore, the identification of resistance mechanisms and the addition of new drugs may better block the development of HER family receptor signals. ${ }^{9}$ Hence, it is particularly important to find new and efficient treatments. Docetaxel (DTX) is a taxol anti-tumor drug that plays an anti-tumor role by interfering with microtubule network necessary for cell mitosis and interphase cell function. ${ }^{10}$ It also has the characteristics of high activity, which can kill tumor cells by increasing the number of microtubules in the body. ${ }^{11}$ At present, DTX has been widely used as an intravenous anti-cancer drug for solid malignant tumors such as non-small cell lung cancer, $\mathrm{BC}$ and prostate cancer. ${ }^{12}$ According to the research of Lee et al, DTX intervention for $\mathrm{BC}$ patients can prevent and reduce the incidence of febrile neutropenia and the hospitalization rate of patients. ${ }^{13}$ Another study reported that TZ combined with DTX (TZ+DTX) is the main drug to treatment HER-2+ $\mathrm{BC},{ }^{14}$ indicating that $\mathrm{TZ}+\mathrm{DTX}$ is feasible for the treatment of HER-2+ BC.

However, there are currently few studies on the combined application of TZ and DTX in HER-2+ BC. Accordingly, TZ +DTX was given to HER-2-positive BC patients in this study, to observe the therapeutic effect and the improvement of serum tumor markers (TMs), and to analyze the risk factors affecting the therapeutic effect, so as to provide more reference for the treatment of HER-2+ BC patients.

\section{Materials and Methods General Data}

The study population of this retrospective study was 96 patients with HER-2+ BC treated in the First Affiliated Hospital of Anhui University Of Science and Technology from January 2019 to December 2020. According to different treatment plans, the patients were divided into two arms, with 48 cases each. The control group (CG) was treated with DTX, and the research group (RG) was treated with TZ+DTX. This study was ethically reviewed and approved by the First Affiliated Hospital of Anhui University Of Science and Technology Ethics Committee, and was conducted in strict compliance with the Declaration of Helsinki. All the subjects and their guardians signed the informed consent to participate. Inclusion criteria: All the enrolled patients were diagnosed as HER-2+ BC by pathology and imaging, ${ }^{15}$ with life expectancy $>6$ months, detailed and complete clinical data, as well as high cooperation with the follow-up. The exclusion criteria were previous chemotherapy with DTX, use of drugs in the past six months that may affect the indicators of this study, other antitumor treatments in the past 1 month, severe blood system diseases, other malignancies, bone marrow dysfunction or heart, and liver and kidney dysfunction. Additionally, dropouts and those who lost to follow up were excluded.

\section{Treatment Methods}

CG: DTX (Sunho Pharmaceutical, Guangdong, China, $\mathrm{H} 20178011)$ was given at $75 \mathrm{mg} \cdot \mathrm{m}^{-2}$, iv $\mathrm{gtt}$, with the infusion time $\leq 1 \mathrm{~h}$. The drug was infused on the first day of the 3-week chemotherapy cycle, and patients were treated for a total of 4 chemotherapy cycles.

RG: TZ (USA, J20090090) was added on the basis of intervention in $\mathrm{CG}$, with the first dose of $8 \mathrm{mg} \cdot \mathrm{kg}^{-1}$, iv gtt, and infusion time $\leq 1.5 \mathrm{~h}$. After that, the dosage was adjusted to $6 \mathrm{mg} \cdot \mathrm{kg}$, and the infusion time was $\leq 1.5 \mathrm{~h}$. $\mathrm{TZ}$ was administered intravenously once every 4 weeks for a total of 4 times.

\section{Outcome Measures}

1. Detection of TMs and inflammatory factors (IFs): ${ }^{16}$ Fasting blood samples $(5 \mathrm{~mL})$ were collected before and after treatment in both groups and centrifuged $\left(1500 \times \mathrm{g}, 4^{\circ} \mathrm{C}\right)$ for $10 \mathrm{~min}$. Serum carcinoembryonic antigen (CEA), carbohydrate antigen (CA)125, CA15-3, C-reactive protein (CRP) and tumor necrosis factor- $\alpha($ TNF- $\alpha)$ were measured before and after treatment strictly according to the instructions of 
Enzyme Linked Immunosorbent Assay (ELISA) kits (Valan Biotechn, Shanghai, China, ABE10309, E01788, E01292, ABE11427, E01593). The samples to be tested were incubated with the coating solution in the ELISA plate at $37^{\circ} \mathrm{C}$ for $2 \mathrm{~h}$ and stayed overnight at $4^{\circ} \mathrm{C}$. They were then closed overnight with $10 \%$ calf serum at $4^{\circ} \mathrm{C}$. After washing, the samples to be tested were cultured with primary antibodies at $37^{\circ} \mathrm{C}$ for $2 \mathrm{~h}$ and then with the secondary antibody at the same temperature for $1 \mathrm{~h}$. After the color reaction, the stopping solution was added and the absorbance of each well was measured at $450 \mathrm{~nm}$ with Multiskan FC microplate (Thermo Fisher Scientific, Shanghai, China).

2. Clinical efficacy: The clinical efficacy after treatment was observed in both groups. If the $\mathrm{BC}$ lesion disappeared completely after treatment and maintained for 4 weeks, it was a complete response $(\mathrm{CR})$. A partial response (PR) was indicated when the size of the $\mathrm{BC}$ lesion was reduced by more than $50 \%$ after treatment for at least 4 weeks. If the reduction of $\mathrm{BC}$ lesion size was between $25 \%$ and $50 \%$ after treatment, with no new lesions, it was considered stable disease (SD). If the BC lesion size increased by more than $25 \%$ after treatment, with the appearance of cardiac lesions, it was considered as progressive disease (PD). Total effective rate $=$ $(\mathrm{CR}+\mathrm{PR})$ cases/total cases $\times 100 \%$.

3. Adverse reactions: A series of adverse reactions occurred during treatment, including grade III-IV myelosuppression, grade III-IV gastrointestinal reactions, nausea and vomiting, liver damage, cardiotoxicity, and peripheral neurotoxicity, were observed and recorded in both groups.

4. Quality of life (QoL): ${ }^{17}$ Patients' QoL after treatment was evaluated by the Karnofsky performance status (KPS) from three dimensions, namely, the ability to carry on normal activity and to work, the ability to care for self, and the condition. On a 100point scale, higher scores indicate better QoL.

\section{Statistical Processing}

SPSS21.0 (SPSS, Inc, Chicago, IL, USA) and GraphPad Prism 6.0 (GraphPad Software Inc., San Diego, CA, USA) were used for data statistical analysis and image rendering, respectively. Counting data were described as number of cases/percentages $[\mathrm{n}(\%)]$, and compared by Chi-square test or continuous correction Chi-square when the theoretical frequency was $<5$. Mean \pm SD was used for measurement data, and $T$-test of independent samples (inter-group) and paired $T$-test (intra-group) were the methods for comparison. The influencing factors of adverse prognosis were analyzed by Logistic regression. A statistically significant difference is defined as $\mathrm{P}<0.05$.

\section{Results \\ General Data}

The general clinical data such as average age, average body mass, residence, ethnicity, education, tumor type, TNM staging, as well as smoking and drinking history were not statistically different between the two arms $(\mathrm{P}>0.05)$, Table 1 .

\section{Comparison of Serum TMs Before and After Treatment}

TMs CEA, CA125 and CA15-3 had no evident difference between RG and $C G$ before treatment $(P>0.05)$; After treatment, the above TMs reduced remarkably in both arms $(\mathrm{P}<0.001)$, and the average levels in $\mathrm{RG}$ were lower than those in $\mathrm{CG}(\mathrm{P}<0.001)$, Figure 1.

\section{Comparison of IFs Before and After Treatment}

The IFs CRP and TNF- $\alpha$ had no significant difference between $\mathrm{CG}$ and $\mathrm{RG}$ before treatment $(\mathrm{P}>0.05)$; After treatment, CRP and TNF- $\alpha$ reduced markedly in both arms $(\mathrm{P}<0.001)$, with more significant reductions in $\mathrm{RG}$ $(\mathrm{P}<0.001)$, Figure 2 .

\section{Comparison of Curative Effects After Treatment}

The Response Evaluation Criteria In Solid Tumors (RECIST) was used to calculate the clinical efficacy of the two groups after treatment. It was found that there were 6 cases of CR, 36 cases of PR, 4 cases of SD and 2 cases of $\mathrm{PD}$ in $\mathrm{RG}$ after treatment, with a total effective rate of $87.50 \%$. In CG, there were 4 cases of CR, 29 cases of PR, 7 cases of SD and 8 cases of PD, and the total effective rate was $68.75 \%$. The total effective rate of $R G$ was significantly higher than that of $\mathrm{CG}(\mathrm{P}<0.05)$, Table 2 .

\section{Adverse Reactions During Treatment}

The statistics of the incidence of adverse reactions during treatment revealed no significant difference between RG and CG $(20.83 \%$ vs $25.00 \%, \mathrm{P}>0.05)$, Table 3 . 
Table I General Data of Patients in Two Groups [n(\%)] (Mean \pm SD)

\begin{tabular}{|c|c|c|c|c|}
\hline Categories & Research Group ( $n=48)$ & Control Group $(n=48)$ & $\mathbf{t} / \chi^{2}$ & $\mathbf{P}$ \\
\hline Average age (years) & $50.16 \pm 5.27$ & $50.39 \pm 5.34$ & 0.212 & 0.832 \\
\hline BMI (kg/m2) & $22.43 \pm 3.12$ & $22.85 \pm 3.17$ & 0.654 & 0.515 \\
\hline Residence & & & 1.046 & 0.307 \\
\hline Urban & $28(58.33)$ & $23(47.92)$ & & \\
\hline Rural & $20(4 I .67)$ & $25(52.08)$ & & \\
\hline Ethnicity & & & 1.688 & 0.194 \\
\hline Han & $35(72.92)$ & $29(60.42)$ & & \\
\hline Ethnic minorities & $13(27.08)$ & $19(39.58)$ & & \\
\hline Educational background & & & 0.667 & 0.414 \\
\hline$\geq$ High school & $22(45.83)$ & $26(54.17)$ & & \\
\hline$<$ High school & $26(54.17)$ & $22(45.83)$ & & \\
\hline Tumor type & & & 0.488 & 0.922 \\
\hline Invasive ductal carcinoma & $24(50.00)$ & $27(56.25)$ & & \\
\hline Invasive lobular carcinoma & $19(39.58)$ & $17(35.42)$ & & \\
\hline Inflammatory breast cancer & $2(4.17)$ & $2(4.17)$ & & \\
\hline Medullary carcinoma & $3(6.25)$ & $2(4.17)$ & & \\
\hline TNM staging & & & 0.274 & 0.872 \\
\hline Illa & $18(37.50)$ & $19(39.58)$ & & \\
\hline Illb & $20(4 I .67)$ & $21(43.75)$ & & \\
\hline IV & $10(20.83)$ & $8(16.67)$ & & \\
\hline History of smoking & & & 0.668 & 0.414 \\
\hline Yes & $27(56.25)$ & $23(47.92)$ & & \\
\hline No & $21(43.75)$ & $25(52.08)$ & & \\
\hline History of drinking & & & 0.389 & 0.533 \\
\hline Yes & $30(62.50)$ & $27(56.25)$ & & \\
\hline No & $18(37.50)$ & $21(43.75)$ & & \\
\hline
\end{tabular}

\section{Comparison of QoL Scores Before and After Treatment}

The QoL assessed by the KPS score showed no distinct difference between $C G$ and $R G$ before treatment $(P>0.05)$; After treatment, the KPS score increased significantly in both arms $(\mathrm{P}<0.001)$, and the increase was more evident in RG $(\mathrm{P}<0.001)$, Table 4.

\section{Multivariate Analysis of Factors Affecting the Curative Effect of Patients with HER-2+ BC}

To evaluate the factors affecting treatment efficacy, the patients were divided into good prognosis group $(n=75)$ and poor prognosis group $(\mathrm{n}=21)$ based on the follow-up results. Multivariate Logistic regression analysis was performed for the factors with differences, and the results identified that CEA ( $\mathrm{P}=0.015), \mathrm{CA} 125(\mathrm{P}=0.014)$, CA15$3(\mathrm{P}=0.008)$ and DTX monotherapy $(\mathrm{P}=0.001)$ were independent risk factors affecting the curative effect of HER-2 $+\mathrm{BC}$ patients. That is to say, the high expression of CEA, CA125 and CA15-3, and DTX monotherapy increase the risk of adverse prognosis, Tables 5 and 6 .

\section{Discussion}

Generally, patients with HER-2+ BC have large tumors and accompany by systemic metastasis, with a poor overall condition. Therefore, combined chemotherapy is the main means to treat the disease. ${ }^{18}$ In recent years, studies have shown that patients with HER-2+ BC will develop severe myelosuppression and serious anemia and hypoxia. ${ }^{19}$ Moreover, given that patients are older with poor tolerance, it is of great significance to choose a treatment scheme with high efficacy and safety. 

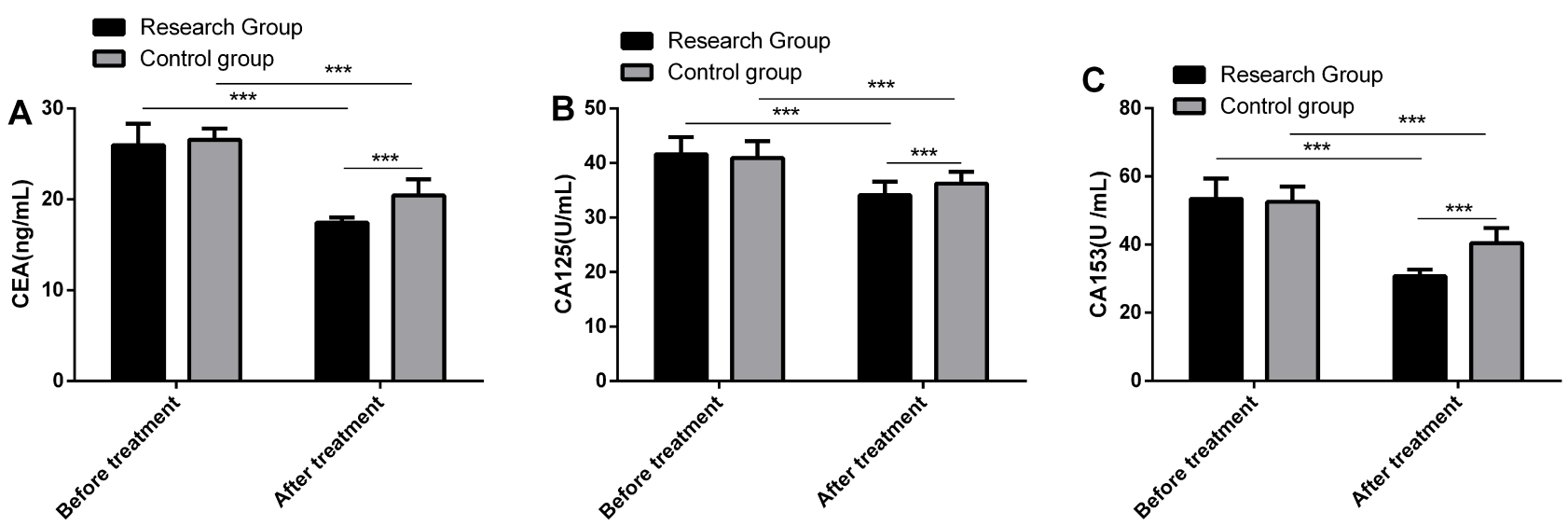

Figure I Comparison of serum tumor markers between two groups before and after treatment. (A) There was no difference in CEA expression between the two groups before treatment, but CEA in the research group was significantly lower than that in the control group after treatment. (B) There was no difference in CAI 25 expression between the two groups before treatment, but CAI25 in the research group was significantly lower than that in the control group after treatment. (C) There was no difference in CAI5-3 expression between the two groups before treatment, but CAI5-3 expression in the research group was significantly lower than that in the control group after treatment.

Note: *** Indicates $\mathrm{P}<0.001$.


Figure 2 Comparison of inflammatory factors between two groups before and after treatment. (A) There was no difference in CRP expression between the two groups before treatment, but CRP in the research group was significantly lower than that in the control group after treatment. (B) There was no difference in TNF- $\alpha$ expression between the two groups before treatment, but TNF- $\alpha$ expression in the research group was significantly lower than that in the control group after treatment. Note: ****Indicates $\mathrm{P}<0.001$.

Studies have shown that the proliferation of cancer cells is an important pathological factor affecting the prognosis and chemotherapy effect of $\mathrm{BC}$ patients. The more the cancer cells proliferate, the higher the content of TMs synthesized by cancer cells and released into the blood circulation. ${ }^{20}$ In order to explore the effect of TZ+DTX on serum TMs in patients with HER-2+ BC, we analyzed related markers. CEA, a traditional tumor-associated antigen, is highly expressed in the serum of patients with advanced cancer, and is often used to assist the diagnosis of lung cancer, BC, liver cancer and other tumors. ${ }^{21}$ CA125 is a specific tumor marker for ovarian and

Table 2 Comparison of Curative Effects Between Two Groups After Treatment [n(\%)]

\begin{tabular}{|l|l|l|l|l|l|l|}
\hline Groups & $\mathbf{n}$ & CR & PR & SD & PD & Total Effective Rate (\%) \\
\hline Research group & 48 & $6(12.50)$ & $36(75.00)$ & $4(8.33)$ & $2(4.17)$ & $42(87.50)$ \\
Control group & 48 & $4(8.33)$ & $29(60.42)$ & $7(14.58)$ & $8(16.67)$ & $33(68.75)$ \\
$\chi^{2}$ & - & - & - & - & - & 4.937 \\
P & - & - & - & - & - & 0.026 \\
\hline
\end{tabular}


Table 3 Incidence of Adverse Reactions in Two Groups During Treatment [n(\%)]

\begin{tabular}{|l|l|l|l|l|l|l|l|l|}
\hline Groups & $\mathbf{n}$ & $\begin{array}{l}\text { Grade III-IV } \\
\text { Myelosuppression }\end{array}$ & $\begin{array}{l}\text { Grade III-IV } \\
\text { Gastrointestinal } \\
\text { Reaction }\end{array}$ & $\begin{array}{l}\text { Nausea } \\
\text { and } \\
\text { Vomiting }\end{array}$ & $\begin{array}{l}\text { Liver } \\
\text { Function } \\
\text { Damage }\end{array}$ & Cardiotoxicity & $\begin{array}{l}\text { Peripheral } \\
\text { Neurotoxicity }\end{array}$ & $\begin{array}{l}\text { Total } \\
\text { Incidence } \\
\text { (\%) }\end{array}$ \\
\hline Research group & 48 & $3(6.25)$ & $2(4.17)$ & $2(4.17)$ & $1(2.08)$ & $I(2.08)$ & $I(2.08)$ & $10(20.83)$ \\
Control group & 48 & $4(8.33)$ & $3(6.25)$ & $3(6.25)$ & $2(4.17)$ & $0(0.00)$ & $0(0.00)$ & $12(25.00)$ \\
$\chi^{2}$ & - & 0.154 & 0.221 & 0.221 & 0.344 & 1.011 & 1.011 & 0.236 \\
P & - & 0.695 & 0.646 & 0.646 & 0.558 & 0.315 & 0.315 & 0.627 \\
\hline
\end{tabular}

Table 4 Comparison of Quality of Life Scores Between Two Groups Before and After Treatment (Mean \pm SD)

\begin{tabular}{|l|c|l|l|}
\hline Groups & \multirow{n}{*}{} & \multicolumn{2}{|l|}{ KPS Score } \\
\cline { 3 - 4 } & & Before Treatment & After Treatment \\
\hline Research group & 48 & $77.62 \pm 5.29$ & $91.05 \pm 8.22$ \\
Control group & 48 & $76.89 \pm 6.47$ & $84.64 \pm 7.12$ \\
t & - & 0.605 & 4.084 \\
P & - & 0.547 & $<0.001$ \\
\hline
\end{tabular}

Table 5 Variable Assignment Table of Unconditional Logistic Regression Analysis

\begin{tabular}{|l|l|l|}
\hline Factor & Variable & Assignment \\
\hline CEA & $X 1$ & Continuous variable \\
CAI25 & X2 & Continuous variable \\
CAI5-3 & X3 & Continuous variable \\
CRP & $X 4$ & Continuous variable \\
TNF- $\alpha$ & $X 5$ & Continuous variable \\
KPS score & X6 & Continuous variable \\
Therapy & X7 & 0 docetaxel, I = trastuzumab combined \\
& & with docetaxel \\
\hline
\end{tabular}

Table 6 Multivariate Analysis of Factors Influencing the Curative Effect of Patients with HER-2+ Breast Cancer

\begin{tabular}{|l|l|l|l|}
\hline Factor & $\mathbf{P}$ & $\mathbf{O R}$ & $\mathbf{9 5 \%} \mathbf{C l}$ \\
\hline CEA & 0.015 & 1.638 & $1.105-2.433$ \\
CAI25 & 0.014 & 1.208 & $0.457-0.998$ \\
CAI5-3 & 0.008 & 1.135 & $0.924-1.458$ \\
CRP & 0.434 & 0.985 & $0.935-1.094$ \\
TNF- $\alpha$ & 0.376 & 0.989 & $0.967-1.014$ \\
KPS score & 0.520 & 1.039 & $0.924-1.169$ \\
Docetaxel monotherapy & 0.001 & 2.172 & $1.785-2.634$ \\
\hline
\end{tabular}

endometrial cancers. ${ }^{22} \mathrm{CA} 15-3$, as a glycoprotein on the cell membrane, is lysed during the proliferation of malignant tumors and enters the blood circulation; It is also a BC-related antigen that can exist in $\mathrm{BC}$ cells and be released into the blood. ${ }^{23}$ According to Chen et al, the detection of serum TMs CEA, CA125 and CA15-3 is widely used in clinical detection of $\mathrm{BC}$ lesions, as well as the occurrence and development of BC. ${ }^{24}$ In this study, the serum levels of TMs CEA, CA125, CA15-3 were detected by ELISA before and after treatment. The results revealed that the levels of the above TMs all decreased remarkably in both arms, especially in RG. It indicates that $\mathrm{TZ}+\mathrm{DTX}$ has a stronger killing effect on BC cells than monotherapy, and can effectively inhibit TMs and slow down tumor progression. Studies have shown that CRP is a sensitive marker of systemic inflammation and is significantly associated with various cancers including BC. ${ }^{25}$ TNF- $\alpha$ is a pleiotropic cytokine that has been found to be mitotic in BC cells both in vivo and in vitro. ${ }^{26}$ In the study of Lemos et al, the use of TZ to treat patients with HER-2+ is shown to adjust the proinflammatory markers in patients to reach the level of healthy controls. ${ }^{27}$ The results of this study showed that after treatment, CRP and TNF- $\alpha$ reduced in both groups and were even lower in CG, which suggested that $\mathrm{TZ}+\mathrm{DTX}$ can reduce the systemic inflammatory response of patients to a certain extent, with more positive therapeutic significance for the control of patients' condition.

Patients with HER-2+ BC are at high risk of recurrence and metastasis and poor overall prognosis, while targeted therapy can significantly reduce the risk of recurrence and death in such patients. ${ }^{28}$ Takahashi et al reported that TZ combined with DTX is effective and well tolerated in patients with recurrent or metastatic HER-2+ BC. ${ }^{29}$ In this study, TZ+DTX was used for intervention. The results revealed that the overall response rate of RG $(87.50 \%)$ was significantly higher than that of CG $(68.75 \%)$, suggesting that the combination therapy was more effective in treating HER-2+ BC. It may be related to the pharmacological mechanism of the two drugs, and their combined application can play a better additive effect, so the curative effect is better. We also 
evaluated the adverse reactions during the treatment. The total incidence of adverse reactions was $20.83 \%$ in $\mathrm{RG}$ and $25.00 \%$ in $\mathrm{CG}$, with no evident difference between the two arms, indicating that TZ+DTX had a high safety and would not increased adverse reactions. With the growing incidence of $\mathrm{BC}$, a large number of women suffer from $\mathrm{BC}$, and the QoL of patients is also increasingly concerned. ${ }^{30}$ In this study, the KPS score was used to evaluate patients' QoL after treatment. It was found that the KPS score increased markedly in both arms after treatment, and was higher in RG compared with $\mathrm{CG}$, demonstrating that TZ+DTX can not only improve the curative effect, but also better improve the QoL of patients. Finally, we analyzed the factors influencing the curative effect of HER-2+ BC patients by Logistic regression. The results identified that high expression of CEA, CA125 and CA15-3, and DTX monotherapy increased the risk of poor prognosis.

To sum up, TZ+DTX can validly improve the clinical efficacy of HER-2+ BC patients and reduce the levels of serum TMs and IFs. However, there is still room for improvement in this study. For example, patients receiving $\mathrm{TZ}$ alone could be enrolled to observe changes in tumor markers during treatment. In addition, we can supplement the basic experiments regarding the treatment mechanism of the two treatments and explore the risk factors affecting the curative effect of patients at the molecular level. In the future, we will gradually improve the study from the above perspectives.

\section{Acknowledgment}

University-level key projects of Anhui University of science and techno logy (NO. ffyyzd2020-03).

\section{Disclosure}

The authors report no conflicts of interest in this work.

\section{References}

1. Kolak A, Kamińska M, Sygit K, et al. Primary and secondary prevention of breast cancer. Ann Agric Environ Med. 2017;24(4):549-553. doi: $10.26444 /$ aaem/75943

2. Shachar S, Hurria A, Muss H. Breast cancer in women older than 80 years. $J$ Oncol Pract. 2016;12(2):123-132. doi:10.1200/ JOP.2015.010207

3. Liu $X, \mathrm{Ma} D, \mathrm{Xu} X$, et al. Genomic landscape and endocrine-resistant subgroup in estrogen receptor-positive, progesterone receptor-negative, and HER2-negative breast cancer. Theranostics. 2018;8(22):6386-6399. doi:10.7150/thno.29164
4. Lin Y, Fu F, Lv J, et al. Identification of potential key genes for HER-2 positive breast cancer based on bioinformatics analysis. Medicine. 2020;99(1):e18445. doi:10.1097/MD.0000000000018445

5. Bhusari P, Vatsa R, Singh G, et al. Development of Lu-177trastuzumab for radioimmunotherapy of HER2 expressing breast cancer and its feasibility assessment in breast cancer patients. Int J Cancer. 2017;140(4):938-947. doi:10.1002/ijc.30500

6. Leung H, Chan A, Muo C, Leung J. Cost-effectiveness of pertuzumab combined with trastuzumab and docetaxel as a first-line treatment for HER-2 positive metastatic breast cancer. Expert Rev Pharmacoecon Outcomes Res. 2018;18(2):207-213. doi:10.1080/ 14737167.2018.1386559

7. Barginear M, John V, Budman D. Trastuzumab-DM1: a clinical update of the novel antibody-drug conjugate for HER2-overexpressing breast cancer. Mol Med. 2013;18:1473-1479.

8. Takahashi M, Takahashi K, Matsumoto S, et al. Low eosinophil percentages as a new predictive marker for infusion reactions due to trastuzumab. Anticancer Res. 2020;40(7):4047-4051.

9. Derakhshani A, Rezaei Z, Safarpour H, et al. Overcoming trastuzumab resistance in HER2-positive breast cancer using combination therapy. J Cell Physiol. 2020;235(4):3142-3156.

10. Hertz D. Exploring pharmacogenetics of paclitaxel- and docetaxel-induced peripheral neuropathy by evaluating the direct pharmacogenetic-pharmacokinetic and pharmacokinetic-neuropathy relationships. Expert Opin Drug Metab Toxicol. 2021;17 (2):227-239. doi:10.1080/17425255.2021.1856367

11. Gaio E, Conte C, Esposito D, et al. Co-delivery of docetaxel and disulfonate tetraphenyl chlorin in one nanoparticle produces strong synergism between chemo- and photodynamic therapy in drug-sensitive and -resistant cancer cells. Mol Pharm. 2018;15 (10):4599-4611.

12. Hendrikx J, Stuurman F, Song J, et al. No relation between docetaxel administration route and high-grade diarrhea incidence. Pharmacol Res Perspect. 2020;8(4):e00633.

13. Lee C, Zhou K, Young W, et al. Febrile neutropenia and its associated hospitalization in breast cancer patients on docetaxel-containing regimen: a retrospective cohort study on duration of prophylactic GCSF administration. Support Care Cancer. 2020;28(8):3801-3812.

14. Rodallec A, Brunel J, Giacometti S, et al. Docetaxel-trastuzumab stealth immunoliposome: development and in vitro proof of concept studies in breast cancer. Int $J$ Nanomedicine. 2018;13:3451-3465.

15. Jin Y, Hua Q, Zheng J, et al. Diagnostic value of ER, PR, FR and HER-2-targeted molecular probes for magnetic resonance imaging in patients with breast cancer. Cell Physiol Biochem. 2018;49 (1):271-281

16. Mu Y, He J, Yan R, Hu X, Liu H and Hao Z. IGF-1 and VEGF can be used as prognostic indicators for patients with uterine fibroids treated with uterine artery embolization. Exp Ther Med. 2016;11:645-649.

17. Frappaz D, Bonneville-Levard A, Ricard D, et al. Assessment of Karnofsky (KPS) and WHO (WHO-PS) performance scores in brain tumour patients: the role of clinician bias. Support Care Cancer. 2021;29(4):1883-1891.

18. Adamo B, Bellet M, Paré L, et al. Oral metronomic vinorelbine combined with endocrine therapy in hormone receptor-positive HER2-negative breast cancer: SOLTI-1501 VENTANA window of opportunity trial. Breast Cancer Res. 2019;21(1):108.

19. Orlando L, Lorusso V, Giotta F, et al. Metronomic oral chemotherapy with cyclophosphamide plus capecitabine combined with trastuzumab (HEX) as first line therapy of HER-2 positive advanced breast cancer: a Phase II trial of the Gruppo Oncologico Italia Meridionale (GOIM). Breast. 2020;53:18-22.

20. Tang J, Luo Y, Tian Z, et al. TRIM11 promotes breast cancer cell proliferation by stabilizing estrogen receptor $\alpha$. Neoplasia. 2020;22 (9):343-351. 
21. Gao Y, Song P, Li H, Jia H, Zhang B. Elevated serum CEA levels are associated with the explosive progression of lung adenocarcinoma harboring EGFR mutations. BMC Cancer. 2017;17(1):484. doi: 10.1186/s12885-017-3474-3

22. Dolscheid-Pommerich R, Keyver-Paik M, Hecking T, et al. Clinical performance of $\mathrm{LOCI}^{\mathrm{TM}}$-based tumor marker assays for tumor markers CA 15-3, CA 125, CEA, CA 19-9 and AFP in gynecological cancers. Tumor Biol. 2017;39(10):1010428317730246.

23. Nam S, Lim W, Jeong J, et al. The prognostic significance of preoperative tumor marker (CEA, CA15-3) elevation in breast cancer patients: data from the Korean breast cancer society registry. Breast Cancer Res Treat. 2019;177(3):669-678.

24. Chen R, Jiang C, Zhu Q, et al. Combining the tumor abnormal protein test with tests for carcinoembryonic antigens, cancer antigen 15-3, and/or cancer antigen 125 significantly increased their diagnostic sensitivity for breast cancer. Medicine. 2020;99(29):e21231.

25. Hong T, Liu A, Cai D, et al. Preoperative serum C-reactive protein levels and early breast cancer by BMI and menopausal status. Cancer Invest. 2013;31(4):279-285.

26. Rivas M, Tkach M, Beguelin W, et al. Transactivation of ErbB-2 induced by tumor necrosis factor alpha promotes NF-kappaB activation and breast cancer cell proliferation. Breast Cancer Res Treat. 2010;122(1):111-124
27. Lemos L, Victorino V, Herrera A, et al. Trastuzumab-based chemotherapy modulates systemic redox homeostasis in women with HER2-positive breast cancer. Int Immunopharmacol. 2015;27 (1):8-14.

28. Abi Jaoude J, de Azambuja E, Makki M, et al. Post-mastectomy radiation therapy in human epidermal growth factor receptor 2 positive breast cancer patients: analysis of the HERA trial. Int $J$ Radiat Oncol Biol Phys. 2020;106(3):503-510.

29. Takahashi M, Ohtani S, Nagai S, et al. The efficacy and safety of pertuzumab plus trastuzumab and docetaxel as a first-line therapy in Japanese patients with inoperable or recurrent HER2-positive breast cancer: the COMACHI study. Breast Cancer Res Treat. 2021;185 (1):125-134.

30. Konieczny M, Cipora E, Roczniak W, Babuśka-Roczniak M, Wojtaszek M. Impact of time to initiation of treatment on the quality of life of women with breast cancer. Int J Environ Res Public Health. 2020;17(22):8325. doi:10.3390/ijerph17228325

\section{Publish your work in this journal}

Cancer Management and Research is an international, peer-reviewed open access journal focusing on cancer research and the optimal use of preventative and integrated treatment interventions to achieve improved outcomes, enhanced survival and quality of life for the cancer patient.
The manuscript management system is completely online and includes a very quick and fair peer-review system, which is all easy to use. Visit http://www.dovepress.com/testimonials.php to read real quotes from published authors. 